\title{
Das Patentgesetz von 1877 - Entstehung und wirtschaftsgeschichtliche Bedeutung
}

\section{Von Rudolf Boch}

Am 25. Mai 1877 wurde im Deutschen Reichstag das Reichspatentgesetz verabschiedet. Dieses Gesetz legte die Rechtsgrundlage für die Entwicklung eines modernen Erfindungsschutzes nicht nur in Deutschland, sondern auch in Nord- und Mitteleuropa und war nicht nur in rechtsgeschichtlicher, sondern auch in wirtschafts- und technikgeschichtlicher Perspektive von epochemachender Bedeutung. Es markiert die Abwendung des unlängst gegründeten Deutschen Reiches vom bis dahin tonangebenden Freihandelsliberalismus und den Beginn einer neuen Epoche aktiver Wirtschaftspolitik des Staates. Zugleich förderte das Patentgesetz die Herausbildung einer spezifischen deutschen Technikkultur, die bis zur Wende zum 20. Jahrhundert aus dem Handelszeichen „Made in Germany“, welches das britische Parlament noch 1887 als Warnung vor billigen deutschen Nachahmungen eingeführt wissen wollte, ein Markenzeichen für Qualitätsproduktion und Produktinnovation werden ließ. Das Gesetzeswerk erwies sich schließlich in der Perspektive der Rechtsgeschichte als zukunftsorientiert, weil es die zunehmende Industrialisierung des Erfindens voraussah.

Wichtige Vorarbeiten für dieses Gesetzeswerk bis hin zur weitgehenden Ausformulierung des Gesetzentwurfs von 1876 wurden von dem damaligen Chemnitzer Oberbürgermeister Wilhelm André als sachkundigem juristischen Berater geleistet.

Ein Jahr nach Gründung des Kaiserlichen Patentamts in Berlin 1878 wurde Chemnitz eine der ersten Patentauslegestellen in Deutschland. In den folgenden Jahrzehnten lag die Zahl der Patentanmeldungen in Chemnitz dann stets weit über dem Durchschnitt vergleichbarer Städte im Reich. 
Diese historischen Ereignisse und die besondere Rolle, die der damalige Oberbürgermeister André spielte, gaben im Jahr 1997, zum 120. Jahrestag der Gesetzgebung, den Anlass für eine von der Technischen Universität Chemnitz, unter meiner Leitung organisierte und durch die Stadt Chemnitz finanzierte Tagung zum Thema "Patentschutz und Innovation in Geschichte und Gegenwart“.

Einerseits warfen Historiker der TU Chemnitz gemeinsam mit auswärtigen Fachkollegen einen Blick zurück auf die lange Vorgeschichte und die Entstehungszusammenhänge des gesetzlichen Erfinderschutzes. Auch wurden die weitere Entwicklung der Patentgesetzgebung im 20. Jahrhundert und die Folgewirkungen des Patentwesens auf technologische Kultur und industrielle Innovationsabläufe thematisiert.

Andererseits sollte das historische Ereignis Ausgangspunkt eines Brückenschlags zur Gegenwart werden, bot die Tagung mithin ein Forum, um „interdisziplinär“ mit Unternehmensvertretern, Wirtschaftswissenschaftlern, Industriesoziologen, Mitarbeitern des Deutschen Patenamts und des Bundesforschungsministeriums über die Probleme und Chancen von Erfinderschutz und technischer Innovation an der Wende zum 21. Jahrhundert zu diskutieren. Schon in den 1990er Jahren gab es mithin das absichtsvolle Bemühen Wilhelm André im Gedächtnis unserer Stadt zu behalten und zugleich an die bedeutende Industriegeschichte von Chemnitz zu erinnern, nicht zuletzt um die aufkeimende Hoffnung auf eine erneuerte ökonomische Prosperität dieser Stadt zu bestärken.

Lassen Sie mich - dreizehn Jahre danach - im kleineren Rahmen der heutigen Veranstaltung noch einmal in knapper Form auf die Entstehung und wirtschaftsgeschichtliche Bedeutung des Gesetzes eingehen: Das Patentgesetz von 1877 steht nicht nur am Anfang einer Epoche 
zunehmender staatlicher Intervention im Deutschen Kaiserreich, es ist auch eine beredtes Beispiel dafür, daß in einem Staat, der sich bereits auf dem besten Weg zu einem Industriestaat befand, staatliches Handeln nicht mehr allein Ausdruck reinen Staatswillens sein konnte, sondern bereits erheblicher Einflußnahme von organisierten Interessen ausgesetzt war. Das Entstehen des Patentgesetzes ist ein frühes Exempel dafür, wie in verhältnismäßig kurzer Zeit eine - modern ausgedrückt - „pressure group“ entsteht, die einen Stimmungswandel in Ministerien und öffentlicher Meinung herbeiführt oder zumindest einen sich abzeichnenden Stimmungswandel nachhaltig unterstützt und diesen dann rasch in eine legislative Form gießen kann. ${ }^{1}$ Kern dieser 'pressure group' wurde der „Deutsche Patentschutz-Verein“ unter dem Vorsitz von Werner Siemens.

Der Umschwung der öffentlichen Meinung geschah innerhalb von nur fünf Jahren. Noch um 1870 schien der Patentschutz in Deutschland keine Zukunft mehr zu haben. Seit dem Sommer 1868 lag dem Reichstag des Norddeutschen Bundes ein offiziell durch Bismarck gestellter Antrag vor, der auf die völlige Abschaffung des Erfindungsschutzes zielte. „Spiritus rector“ dieses Antrages war freilich nicht Bismarck selber, sondern Rudolf Delbrück (1817 - 1903), die wohl bedeutendste Beamtenpersönlichkeit der 1850er bis 70er Jahre. Delbrück seit 1867 schließlich einflußreicher Präsident des Norddeutschen Bundesamtes (und späteren Reichskanzleramtes), war zutiefst überzeugter Wirtschaftsliberaler und Freihändler.

Spätestens seit dem 6. Kongreß Deutscher Volkswirte in Dresden im Jahre 1863, der sich vorrangig mit dem Patentwesen beschäftigte, war die Abschaffung jeglichen Patentschutzes zu einem vereinheitlichten Ziel der Freihandelsbewegung geworden. Erfindungsschutz wurde von diesem Kongreß als ein der freien Konkurrenz abträgliches Gewerbemonopol gebrandmarkt, als Relikt aus merkantilistischer Zeit. Auch die Idee des geistigen Eigentums wurde in Dresden grundsätzlich verworfen, da - so die Begründung - jede Idee eines 
Einzelnen Ausfluß des gesamtgesellschaftlichen Wissensstandes wäre, so daß jederzeit mehrere Personen die gleiche Idee haben könnten - was viele Doppelerfindungen beweisen würden.

Da die - noch eine Generation zuvor unvorstellbare - Rasanz der technischen Entwicklung die inhaltlichen Bestimmungen fast aller bestehenden Patentregelungen längst überholt hatte und sich der preußische Behördenapparat für die technische Vorprüfung bei Patentanmeldungen als hoffnungslos überfordert erwies, hatte die Freihandelsschule nicht nur die ökonomische Theorie, sondern auch den realen Erfahrungshorizont zahlreicher Unternehmer auf ihrer Seite. Eine Umfrage des preußischen Handelsministeriums unter den Handelskammern im selben Jahr 1863 bestätigte das: 31 Handelskammern stimmten für die ersatzlose Aufhebung des Patentschutzes; nur 16 Kammern plädierten für die prinzipielle Beibehaltung, forderten aber mit Nachdruck eine Reform sowie eine zukünftige Rechtsvereinheitlichung auf dem Gebiet des Zollvereins. ${ }^{2}$

Mit der grundsätzlichen Infragestellung des Erfindungsschutzes seit Anfang der 1860er Jahre erhoben sich vermehrt Gegenstimmen, aber es verbietet sich m. E. bereits von einer „Propatentbewegung“ zu sprechen. Eine Plattform fanden die Patentbefürworter im 1856 gegründeten „Verein Deutscher Ingenieure“ (VDI), der seit 1861 mit zahlreichen Denkschriften und Petitionen hervortrat.

Erst als an der Wende von den 1860er zu den 1870er Jahren die letzte Stunde des Erfindungsschutzes gekommen schien, kam es zu einer massiven Zunahme von öffentlichen Interventionen aus Unternehmerkreisen für die Fortführung und Vereinheitlichung des Patentschutzes. Man kann fast von einer Konfrontation der Erfinderunternehmer der Elektro-, Maschinenbau- und Chemischen Industrie mit tonangebenden Nationalökonomen und dem - 
häufig indifferenten -, die damaligen Handelskammern noch stark dominierenden Typus des Kaufmannsunternehmers sprechen.

Nicht zuletzt der Krieg gegen Frankreich hatte die Beratungen über die Abschaffung des Patentschutzes in den Gremien des Norddeutschen Bundes 1870 zum Erliegen gebracht. Die Reichseinigung von 1871 drängte nun erneut auf eine Entscheidung hin: entweder ein einheitlicher Patentschutz für das gesamte Reichsgebiet oder die konsequente Aufhebung des Erfindungsschutzes wie in den Niederlanden. Auch das gewachsene internationale Gewicht des neuen Kaiserreiches, ließen die Frage des Patentschutzes wieder an Aktualität gewinnen.

Dieser internationale Aspekt der Patentfrage begann sich nun zugunsten der Patentbefürworter auszuwirken. So galt es seit 1872 als ausgemacht, daß Großbritannien als die größte Industriemacht der Welt, nach langen eingehenden Beratungen am Erfindungsschutz festhalten würde. Auch der Internationale Patentschutzkongreß von 1873, am Rande der Wiener Weltausstellung abgehalten, und maßgeblich von den Gebrüdern Siemens initiiert, machte die Gefahr einer Isolierung des jungen Reiches deutlich; hatte der unmittelbare Anlaß dieses Kongresses doch darin bestanden, daß sich führende amerikanische Firmen wegen des mangelhaften preußischen Patentschutzes geweigert hatten, ihre technischen Innovationen auszustellen. ${ }^{3}$

Aber wichtige Protagonisten des Patentschutzes erkannten sehr klar, daß das Argument der internationalen Isolierung nicht ausreichte, den Patentschutz gesetzlich zu sichern, so lange die Patentgegner in der durch Preußen hegemonisierten Administration des Reiches noch derart stark waren und in den liberalen Parteien des Reichstages noch über eine erhebliche Anhängerschaft verfügten. In einem Brief an den Kölner Ingenieur und Unternehmer Eugen Langen, den die Forschung als „Geburtsurkunde des Deutschen Patentschutzvereins“ 
bezeichnet hat, ${ }^{4}$ schrieb Werner Siemens im Februar 1874: „Ich bin der Ansicht, daß die Frage für parlamentarische Behandlung noch nicht reif ist.... Wir müssen den Beweis führen, daß nicht mehr nur einige Erfinder um Schutz schreien, sondern daß wirklich achtungsgebietende Klassen und Interessen ihn fordern.“

Ende März 1874 trafen sich Werner Siemens, Wilhelm André (1827 - 1903), der Dresdener Ingenieur Carl Pieper (1838 - 1908) und andere in Berlin, um die Vereinsgründung vorzubereiten und schon zwei Monate später konnte Siemens seinem Bruder Wilhelm in London die Erfolgsmeldung geben: „Die ganze deutsche Großindustrie und wissenschaftliche Technik ist im Verein vertreten, und eine Menge Vereine haben ihren Betritt mit ansehnlichen Beiträgen zugesagt. “5 Siemens gewann darüber hinaus auch erstmals Unterstützer in der Ministerialbürokratie.

Es war aber nicht in erster Linie das Resultat einer geschickten Lobby-Politik und auch nicht nur die Verunsicherung der Berliner Bürokratie durch die unerwartet zähe Wirtschaftskrise, sondern die Argumentationsweise von Werner Siemens, seine beeindruckende Krisenanalyse in Verbindung mit der Forderung nach Patentschutz, die die patentfeindliche Stimmung umschlagen ließ. Ich möchte deshalb diese Analyse des Wirtschaftsstandorts Deutschland an der Schwelle zur Hochindustrialisierung und Siemens Vorschläge für eine neue technologische Kultur in den Grundzügen knapp skizzieren:

Für Siemens war die Wirtschaftskrise nach 1873 keine einfache Handels- oder $\underline{\text { Konjunkturkrise, }}$ sondern eine sich schon länger anbahnende Strukturkrise der industriellen Produktion in Deutschland, nicht zuletzt eine Krise der Technologiekultur. Der Aufschwung der deutschen Industrie in den 1850er und 60er Jahren war - folgt man Siemens - durch zwei Kostenvorteile begünstigt worden: durch die reine Nachahmung ausländischer Innovationen 
in den meisten Industriezweigen waren Investitionskosten für Forschung und Entwicklung weitgehend entfallen. Durch diese Ersparnis und die sehr niedrigen deutschen Löhne hatten deutsche Waren - trotz geringerer Produktivität - häufig billiger angeboten werden können als ausländische. Diese notorische Nachahmungspraxis hätte den deutschen Produkten im Ausland aber den Ruf „Billig und Schlecht“ eingetragen und zu Exportverlusten geführt, während die Streikwellen von 1871 bis 1873 und die erheblichen Lohnsteigerungen in ihrem Gefolge diese Billigexporte zusätzlich erschwert hätten.

Das Lohnniveau werde langfristig weiter steigen und eine Rückeroberung fremder Märkte sei nur dadurch möglich, daß die Warenqualität englischen Standard erreiche oder diesen übertreffe. Eine Grundvoraussetzung dafür sei - so Siemens - ein industrielles Leistungsdenken, welches nur entstehen können, wenn die gesellschaftliche Anerkennung der

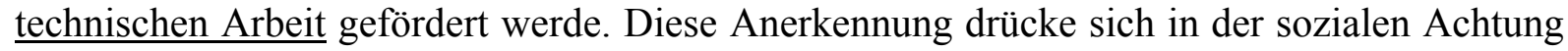
der Techniker und Unternehmer und im Schutz der geistigen Arbeit und deren Belohnung durch ein Patent aus.

Erfindertätigkeit beruhe außerdem nicht auf jenem „mühelosen Einfall“ - so Siemens - , wie ihn die Freihandelsschule behauptet hatte, sondern die Umsetzung einer Idee in die Praxis erfordere Kapital und Arbeit; die hierdurch bewirkte Förderung des technischen Fortschritts und des wirtschaftlichen Wachstums rechtfertige wegen des „gesellschaftlichen Nutzens“ das Patent qua Belohnung und weiteren Ansporn der technischen Aktivität. Technischer Fortschritt werde außerdem - so argumentierte Siemens ganz modern - durch Unternehmen zunehmend bewußt induziert, d. h. technische Problemlösungen würden gesucht, weil wirtschaftliche Notwendigkeiten dies geraten erscheinen ließen. Dem Patent falle hierbei die Aufgabe zu, diesen Prozeß rechtlich gegen die Konkurrenz abzusichern. Die steigende 
Gewinnerwartung mindere das Investitionsrisiko einer Neuerung und steigere die Investitionsbereitschaft des Unternehmers.

Nationale Töne waren Siemens nicht fremd. Trotz seiner vielfältigen internationalen Geschäftsaktivitäten dachte auch er in den Kategorien der Stärkung der deutschen Industrie als Basis für eine starke deutsche Nation. Siemens stand dann auch den nach 1879 von wichtigen Industriezweigen immer lauter geforderten Schutzzöllen nicht grundsätzlich ablehnend gegenüber. Trotzdem hat er als Person den Patentschutz - das sollte hervorgehoben werden - $\underline{\text { nicht }}$ als ein Instrument zur Marktabschottung oder als flankierende Maßnahme einer umfassenden Schutzzollpolitik angepriesen. Patentschutz war für ihn zwar ein Hebel zur Verbesserung der Produktqualität, aber nicht einfach „Waffe im Handelskrieg“. Das sahen einflussreiche deutsche Industrielle, die mit Siemens nach 1873 zunächst die internationale Kompatibilität eines zukünftigen deutschen Patentschutzes vorangetrieben hatten, mit zunehmender Dauer der krisenhaften Wirtschaftsentwicklung freilich anders. Sie verhinderten mit Unterstützung der Ministerialbürokratie den Beitritt Deutschlands zur „Internationalen Union zum Schutz des gewerblichen Eigentums“ bis 1901.

Auf der Grundlage des Gesetzentwurfes des „Patentschutzvereins“ ernannte das Kanzleramt im September 1876 eine durchweg mit Patentbefürwortern besetzte Patent-Kommission, die ein Reichsgesetz vorbereiten sollte. Ende Mai 1877 nahm ein mehrheitlich eigentlich noch freihändlerisch gestimmter Reichstag die Gesetzesvorlage an. Das glatte Passieren der parlamentarischen Hürden läßt sich auch damit erklären, daß das Gesetz ,als eine vorläufige politische Konzession an die notleidende Industrie gedacht war. ${ }^{\text {‘6 }}$ Der zentralen Forderung der Schwerindustrie sowie der einflußreichen Landwirtschaft, der Einführung von hohen Schutzzöllen als Markteintrittsbarriere für die ausländische Konkurrenz, verschloß sich die Reichstagsmehrheit noch. Die Revision der bisherigen Zollpolitik erfolgte erst 1879. 
Das Patentgesetz wird heute als ein erster Schritt hin zu einer aktiven Wirtschaftspolitik des Deutschen Kaiserreichs bewertet, es war zugleich auch ein erster Schritt vieler von der Freihandelsschule beeinflußter liberaler Abgeordneter, deren Blütenträume zerstoben waren, den wirtschaftlichen Akteuren, den Unternehmern und ihrer Sicht der Dinge - jenseits vorgefertigter Lehrmeinungen - zuzuhören. In ihre Bereitschaft die Krisenanalyse eine Siemens aufzunehmen, mischte sich aber eine kräftige Dosis des alten freihändlerischen Mißtrauens gegenüber den Ansprüchen des Erfinders. Im Kräfteparallelogramm von industriellen Interessen und Geringschätzung der individuellen Erfinderleistung gestaltete sich das deutsche Patentgesetz von 1877 der Tendenz nach einzelerfinderfeindlich. Vor allem der Ausführungszwang ( $§ 11)$, die progressiven Patentgebühren bis zu einer Höhe von 5.000 Mark (§ 8), aber auch das sog. Anmelderprinzip (§ 3) führten in der Folgezeit, als auch das Problem der Arbeitnehmererfindungen immer gewichtiger wurde, zu ständig wachsender Kritik.

Hat nun das Patentgesetz den Aufschwung der Erfindertätigkeit, eine neue technologische Kultur im Deutschen Kaiserreich und das damit hoffnungsvoll verknüpfte erneute wirtschaftliche Wachstum ermöglicht oder gefördert?

Ohne Zweifel kann man nach 1877 eine rasch steigende Zahl von Patentanmeldungen und Patenterteilungen beobachten; ein Zeichen von verstärkter technologischer Aktivität. Sicherlich förderte das Patentgesetz auch jene Entwicklung von Technik, Leistungsdenken und Arbeitsstolz, die bis zur Jahrhundertwende aus dem Handelszeichen „Made in Germany“, ein Markenzeichen für Qualitätsproduktion und Produktinnovation werden ließ. ${ }^{7}$ Den wirtschaftlichen Aufstieg Deutschlands bis zum Ersten Weltkrieg vor allem oder gar 
monokausal aus dem erfolgreichen Patentwesen erklären zu wollen, verbietet sich freilich von selbst.

Der quantitative wie qualitative Beitrag von patentierten Erfindungen zum Wirtschaftswachstum ist für die deutsche Volkswirtschaft oder auch nur für einen Industriezweig bisher von der wirtschaftsgeschichtlichen Forschung noch nicht eingehend untersucht worden.

Die Korrelation zwischen steigenden Patentzahlen und Wirtschaftswachstum ist freilich zumindest ein klares Indiz für den positiven Nutzen des neuen, vereinheitlichten Patentschutzes; das wurde auch von den zeitgenössischen Experten so gesehen. Von nur 190 Patenten im Jahre 1877 sprang die Zahl auf über 4.000 erteilte Patente im Jahre 1890. Als im Jahre 1891 das Patentgesetz nach den Vorstellungen der Chemischen Industrie novelliert wurde und auch Stoffpatente zugelassen wurden, weil die Chemieindustrie bewußt neue Stoffe suchte und nicht mehr nur das Verfahren, sondern auch das Produkt selber geschützt haben wollte, stieg die Zahl sofort auf 5.500 Patente. ${ }^{8}$ In den letzten fünf Jahren vor dem Ersten Weltkrieg wurden im Durchschnitt jährlich über 12.000 Patente zugesprochen.

Die Verteilung der Patente über die Industriezweige in der Periode zwischen 1877 und 1914 mag vielleicht noch etwas aufschlußreicher sein, als die absoluten Zahlen der Patenterteilungen. Den Löwenanteil der Patente ging weder an die Chemische Industrie noch an die Elektrotechnische Industrie, die in der wirtschaftshistorischen Forschung gemeinhin als die innovativsten Industriezweige im Kaiserreich gelten, sondern an die Metallverarbeitende Industrie, speziell an den Maschinenbau. $1877 / 78$ wurden fast 40 Prozent aller Patente im Deutschen Reich für den Bereich der Metallverarbeitung (sogar unter Ausschluß des Dampfmaschinenbaus, des Fahrzeugbaus und des elektrischen Anlagenbaus) vergeben, 
während die Chemieindustrie nur 4 Prozent und die Elektroindustrie nur 1 Prozent aller Patente erhielt. 1913 führte die Metallverarbeitende Industrie immer noch mit 32,5 Prozent, während der Anteil der Chemischen Industrie auf 11 Prozent und der Anteil der Elektrotechnischen Industrie auf 8,4 Prozent gestiegen war.

In Relation zur relativen Größe der Industrie lag die Zahl der erteilten Patente für die Chemieindustrie aber weit über den Durchschnitt, beschäftigte sie doch nur 2,5 Prozent aller industriell Beschäftigten im Jahre 1913. ${ }^{9}$

Dieser Vergleich sehr kruder Strukturdaten kann natürlich nur begrenzte Aufschlüsse vermitteln. Der Berliner Wirtschaftshistoriker Wolfram Fischer hat schon vor geraumer Zeit darauf hingewiesen, daß man über den Zusammenhang von Patenterteilung und Wirtschaftswachstum mehr herausbekommen könne, wenn man Patentzahlen und Wachstum in kleineren Brancheneinheiten als der monströsen Metallverarbeitenden Industrie untersuchen würde. Eine zukünftige Forschung muß - so Fischer - dabei den Zeitfaktor, den time-lag zwischen einer Erfindung und ihrem ökonomischen Effekt berücksichtigen und auch Methoden entwickeln, um die ökonomischen Bedeutung eines Patents zu gewichten. 
1 Das betont nachdrücklich Wilhelm Treue in seinem Aufsatz, Die Entwicklung des Patentwesens im 19. Jahrhundert in Preußen und im Deutschen Reich, in: Wissenschaft und Kodifikation des Privatrechts im 19. Jahrhundert, Bd. 4, hrsg. von. Helmut Coing/Walter Wilhelm, Frankfurt a. M. 1979, S. 163 - 182. Seine Beurteilung des Patentgesetzes als großbürgerliches unternehmerfreundliches Komplott erscheint dem Vf. überzogen. Zum derzeitigen Forschungsstand vgl. Margrit Seckelmann, Industrialisierung, Internationalisierung und Patentrecht im Deutschen Reich, 1871 - 1914, Frankfurt a. M. 2006.

${ }^{2}$ Alfred Hagen, Erfinderschutz und Industrialisierung in Preußen 1793 - 1877, Göttingen 1975, S. 81.

${ }^{3}$ Karl-Heinz Manegold, Der Wiener Patentschutzkongreß von 1873. Seine Stellung und Bedeutung in der Geschichte des deutschen Patentwesens im 19. Jahrhundert, in: Technikgeschichte 38 (1971), S. 158 - 165, hier S. 159.

${ }^{4}$ Vgl., ders., Vom Erfindungsprivileg zum „Schutz der nationalen Arbeit““, in: Zeitschrift der TU Hannover 1976/2, S. 13.

${ }^{5}$ Conrad Matschoß, Werner Siemens, Berlin 1916, Bd. 1, S. 521.

${ }^{6}$ Marcel Silberstein, Erfindungsschutz und merkantilistische Gewerbeprivilegien, Winterthur 1961, S. 283.

${ }^{7}$ Der vom Londoner Parlament beschlossene Merchandise Marks Act schrieb vor, daß aus Deutschland nach Großbritannien eingeführte Waren die Herkunftsbezeichnung „Made in Germany“ tragen mußten.

${ }^{8}$ Dazu grundlegend: Arndt Fleischer, Patentgesetzgebung und chemisch-pharmazeutische Industrie im Deutschen Kaiserreich (1871 - 1918), Stuttgart 1984. 
${ }^{9}$ Alle Zahlen nach: Wolfram Fischer, The Role fo Science and Technology in the Economic Development of Modern Germany, in: Science, Technology and Economic Development, hrsg. von Walter Beranek/Georg Ranis, New York u. a. 1978, S. 71 - 113, hier S. 95f.

Weiterführende Literatur zum Thema Patentschutz:

Rudolf Boch (Hg.), Patentschutz und Innovation in Geschichte und Gegenwart, Frankfurt a. M./New York 1999; Kees Gispen, Poems in Steel. National Socialism and the Politics of Inventing from Weimar to Bonn, New York/Oxford 2002. 